\title{
Acute Severe Pancreatitis: A Dreadful Complication of Sodium Valproate
}

\author{
Juhi Gupta ${ }^{1} \cdot$ Rahul Sinha $^{1} \cdot$ Prashant Jauhari $^{1} \cdot$ Rohan Malik $^{2} \cdot$ Biswaroop Chakrabarty $^{1} \cdot$ Sheffali Gulati ${ }^{1}$
}

Received: 13 January 2019 / Accepted: 3 April 2019 / Published online: 24 April 2019

(C) Dr. K C Chaudhuri Foundation 2019

To the Editor: Acute pancreatitis is a rare diagnosis in pediatrics. Valproic acid has been known to trigger this catastrophe in occasional young patients as an idiosyncratic reaction [1]. We describe a typically developing eight-year-old boy who presented to emergency department with acute abdomen (severe, diffuse non-radiating abdominal pain with non-bilious vomiting) and fever for $48 \mathrm{~h}$. He was on oral valproate $(30 \mathrm{mg} / \mathrm{kg} / \mathrm{d})$, phenobarbitone $(3 \mathrm{mg} / \mathrm{kg} / \mathrm{d})$ and clonazepam $(0.04 \mathrm{mg} / \mathrm{kg} / \mathrm{d})$ for his underlying epilepsy. He was diagnosed with super-refractory status epilepticus of unknown etiology nine months back and has been receiving antiepileptics since then. There was not any history of recent change in antiepileptic dosages. On examination, there was tachycardia (pulse rate $=120 / \mathrm{min}$ ), tachypnea (respiratory rate $=$ $38 / \mathrm{min}$ ), diffuse abdominal tenderness and guarding. His hemogram revealed leukocytosis $(12,760 /$ cumm with $60 \%$ neutrophils) and low platelet count (118,000/cumm). Ultrasound abdomen and chest revealed mild ascites and bilateral minimal pleural effusion with non-visualization of pancreas. A working diagnosis of valproate induced acute pancreatitis was considered. Raised serum amylase (647 $\mathrm{SU}$, normal $=28-100 \mathrm{SU}$ ) and serum lipase (514 SU, normal $=0-190 \mathrm{U} / \mathrm{L}$ ) confirmed the presence of pancreatitis. Serum Valproic acid (VPA) level was within therapeutic range $(56.68 \mathrm{mcg} / \mathrm{ml}$, normal $=50-100 \mathrm{mcg} / \mathrm{ml})$. Work up for infective etiology (dengue, rickettsia, chikungunya, coxsackie, cytomegalovirus) was negative. Child was made nil-per-oral and started on intravenous hydration with antibiotics (piperacillin tazobactam and metrogyl), proton pump

Prashant Jauhari

pjauhari0@gmail.com

1 Child Neurology Division, Department of Pediatrics, All India Institute of Medical Sciences, New Delhi 110029, India

2 Division of Pediatric Gastroenterology, Hepatology and Clinical Nutrition, Department of Pediatrics, All India Institute of Medical Sciences, New Delhi, India inhibitors and opioids for pain relief. VPA was immediately stopped and replaced with levetiracetam. On day 2 of hospitalization he developed hypoxia with increased pleural effusion, ascites and loose stools which responded to conservative management within $48-72 \mathrm{~h}$. Child did not have any neurological issues during the stay and was discharged after $1 \mathrm{wk}$. Amylase levels got normalized ( $0.5 \mathrm{SU})$.

In any child on valproate therapy presenting with acute abdomen, pancreatitis should be suspected and investigated. The underlying mechanism of valproate induced pancreatitis is elucidative and may possibly be associated with free radical injury [2]. Pancreatitis can develop after weeks to years of initiation of VPA [3]. Majority of children will respond to timely withdrawal of valproic acid and conservative management [4]. However, occasional fatality is also reported [5]. Pediatricians should be aware of this sinister adverse reaction of VPA.

\section{Compliance with Ethical Standards}

Conflict of Interest None.

\section{References}

1. Pellock JM, Wilder BJ, Deaton R, Sommerville KW. Acute pancreatitis coincident with valproate use: a critical review. Epilepsia. 2002;43:1421-4.

2. Underwood TW, Frye CB. Drug-induced pancreatitis. Clin Pharm. 1993;12:440-8.

3. Werlin SL, Fish DL. The spectrum of valproic acid-associated pancreatitis. Pediatrics. 2006;118:1660-3.

4. Cofini M, Quadrozzi F, Favoriti P, Favoriti M, Cofini G. Valproic acid-induced acute pancreatitis in pediatric age: case series and review of literature. G Chir. 2015;36:158-60.

5. Evans RJ, Miranda RN, Jordan J, Krolikowski FJ. Fatal acute pancreatitis caused by valproic acid. Am J Forensic Med Pathol. 1995;16:62-5.

Publisher's Note Springer Nature remains neutral with regard to jurisdictional claims in published maps and institutional affiliations. 